\title{
Influencia del sistema start-stop en el vehículo respecto durabilidad de la batería de acumuladores
}

\section{Influence of the start-stop system on the vehicle with regard to the durability of the accumulator battery}

\author{
Leonidas Quiroz Erazo ${ }^{1}$, Jhonatan Aguilar Santamaría ${ }^{2}$ Cristhian Rumiguano Jiménez ${ }^{3}$, Germán Erazo Laverde ${ }^{4}$ \\ ${ }^{1}$ Universidad de las Fuerzas Armadas ESPE, Departamento de Energía y Mecánica
}

Correspondencia Autores: laquiroz@espe.edu.ec ${ }^{1}$, jaaguilar6@espe.edu.ec ${ }^{2}$, cdrumiguano@espe.edu.ec ${ }^{2}$, wgerazo@espe.edu.ec ${ }^{3}$

Recibido: 19 de noviembre 2018, Publicado: 18 de diciembre de 2018

\begin{abstract}
Resumen - En la presente investigación se muestra los cálculos realizados para prever como afecta la instalación del sistema start-stop a la batería del vehículo Hyundai Getz 1.6, para seleccionar una diferente o mantener la misma, así como los resultados de las pruebas realizadas con el sistema en funcionamiento con el vehículo en neutro siguiendo los parámetros de pruebas del ciclo europeo de conducción, NEDC, del cual se obtuvo el tiempo de duración de la prueba y el número de paradas que ocurren. Los cálculos se realizaron tomando en cuenta parámetros tales como el consumo de corriente por parte del motor de arranque durante su activación y el amperaje que requieren los componentes electrónicos que hacen funcionar al sistema. Para esta investigación no se tomaron en cuenta los datos de consumo de combustible ni los niveles de emisiones de los gases de escape.
\end{abstract}

Palabras clave- Start-stop, Batería, Sistema de carga, Consumo de corriente.

Resumen- The present investigation shows the calculations made to anticipate the way the start-stop system instalation affect the Hyundai Getz 1.6 vehicle's battery, to select a different one or hold the same, as well as, the test results of the system working with the vehicle in neutral, following the Europe driving test parameters, NEDC, from wich the duration test time and the number of engine stops were obtained. The calculations were made taking into account parameters like the starter motor electric current consumption during its activation and the amperage required by electronics devices that control the system. In this investigation, the gas consumption and the exhaust gases emissions level, were not taking into consideration.

Keywords - Start-stop, Battery, Charging system, Electric current consumption.

\section{INTRODUCCIÓN}

El sistema star-stop fue diseñado para economizar combustible del vehículo mediante el apagado automático del motor de combustión interna, durante los periodos de fases inactivas cuando no es requerido para realizar tracción, y reiniciándole cuando es requerido [1].

La desactivación del motor por parte del sistema se realiza cortando la alimentación de la batería a las bobinas de encendido, lo que provoca que no exista salto de chispa, apagando el motor,

Por otra parte, cuando se requiere que el motor de combustión vuelva a funcionar es necesario activar el motor de arranque, esta acción conlleva un consumo de corriente que se verá aumentado de acuerdo a la cantidad de veces que se repita este proceso durante el funcionamiento del sistema. Este incremento afecta la capacidad de la batería, que debe aumentar de acuerdo a la corriente consumida por el motor de arranque durante su operación [1]. Cabe destacar que el alternador no proporciona energía al sistema en esta fase, así que no se lo considera para la realización de los cálculos.

Al realizar el cálculo de la corriente que consume el motor de arranque se deben tomar en cuenta las características eléctricas del mismo, que vienen determinadas por la tensión nominal a la cual debe funcionar, la potencia nominal y la potencia máxima absorbida. No toda la potencia absorbida se transforma en energía mecánica, una parte se pierde en el mismo circuito del motor, rozamientos mecánicos y la reacción del inducido, quedando una potencia útil muy reducida, por tanto, la potencia mínima del motor de arranque es igual a la potencia necesaria por el motor térmico, más la consumida por el propio motor eléctrico.

La distancia recorrida por el vehículo en una prueba bajo el ciclo de conducción NEDC es de $11 \mathrm{~km} \mathrm{[1],}$ dentro del cual se considera que existen 14 periodos de ralentí con un tiempo de parada de 340 segundos [2].

\section{MÉTODOS Y MATERIALES}

Se empleó la matematización para determinar, mediante ecuaciones y fórmulas, cuál será el efecto de la instalación del sistema sobre la batería del vehículo. Con el resultado obtenido se utilizó el método analítico para verificar sobre que característica del acumulador 
influyen los valores obtenidos, para finalmente, mediante una investigación en el mercado local de componentes eléctricos automotrices, seleccionar el dispositivo que mejor se adapte al nuevo régimen de trabajo, tanto del vehículo como del sistema.

También se empleó el método empírico para realizar pruebas de rendimiento del nuevo acumulador, mediante el probador de baterías DY2015a, que se muestra en la figura 1, se verificó la caída de voltaje en los bornes durante el arranque en un ciclo simulado con el vehículo detenido, realizando durante 15 minutos un total de 12 paradas de 30 segundos.

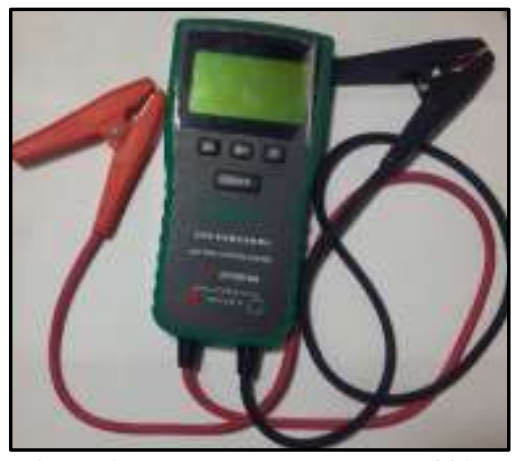

Figura 1: probador de baterías DY2015a

Las características de este equipo son:

- Pruebas en baterías de 12 y 24V.

- Cumple con norma DIN, JIS e inglesas.

- Medición de caída de voltaje en arranque.

- Medición de vida útil de la batería.

- Medición de resistencia interna.

- Prueba del sistema de carga.

En el manual de usuario del probador de baterías también se indica los valores mínimos y máximos de tensión que se debe alcanzar en cada prueba para comprobar si se encuentra en buen estado. Para el caso de las pruebas de arranque, estos valores se muestran en la tabla 1. [4].

Tabla 1: Estado de batería según tensión de arranque.

\begin{tabular}{|c|c|}
\hline Tensión de arranque & Estado \\
\hline $10.7-12.0 \mathrm{~V}$ & Buena \\
\hline $10.2-10.7 \mathrm{~V}$ & Normal \\
\hline $9.6-10.2 \mathrm{~V}$ & No está bien \\
\hline$<9.6 \mathrm{~V}$ & Mal/Dañada \\
\hline
\end{tabular}

Los cables del probador son de color rojo y negro, que van conectados a la terminal positiva y negativa de la batería respectivamente, como se muestra en la figura 2.

Los datos obtenidos de esta prueba se tabularon y compararon entre sí, para verificar como estaba afectando al desempeño de la batería la realización continua de este procedimiento. También se acompañó cada resultado de una observación del comportamiento del vehículo, percibida por las personas que realizaron la prueba.

\section{PRUEBAS Y RESULTADOS}

El cálculo de la potencia mínima necesaria en el motor de arranque parte de la ecuación de par resistente del motor que se expresa en $\mathrm{kgf}^{*} \mathrm{~m}$, con un una cilindrada de 1,61 y un valor de $\mathrm{K}$ igual a 5.

$$
\begin{gathered}
C_{m}=K * V_{t} \\
C_{m}=5 * 1.6 \mathrm{l} \\
C_{m}=6.4 \mathrm{kgf} \cdot \mathrm{m}
\end{gathered}
$$

A partir de este valor se obtuvo la potencia útil o necesaria en el motor eléctrico aplicado al motor térmico para producir el arranque en $\mathrm{kW}$, tomando una velocidad de $150 \mathrm{rpm}$, con la ecuación:

$$
\begin{gathered}
W_{V}=\frac{C_{m} * n}{716.2} * 0.736 \\
W_{V}=\frac{6.4 \mathrm{kgf} \cdot \mathrm{m} * 150 \mathrm{rpm}}{716.2} * 0.736 \\
W_{V}=0.99 \mathrm{~kW}
\end{gathered}
$$

Utilizando un factor de seguridad de 2 y un rendimiento del motor de arranque de 0.8 , se calcula la potencia del arranque con la ecuación:

$$
\begin{gathered}
W_{a}=\frac{A * W_{V}}{\eta} \\
W_{a}=\frac{2 * 0.99 \mathrm{~kW}}{0.8} \\
W_{a}=2.47 \mathrm{~kW}
\end{gathered}
$$

Se halla la potencia mínima necesaria durante el arranque:

$$
\begin{gathered}
W=W_{V}+W_{A} \\
W=(0.99+2.47) k W \\
W=3.46 k W
\end{gathered}
$$


De este valor se puede encontrar la corriente en amperios, que circula por el motor de arranque durante su activación, tomando un voltaje nominal de $12 \mathrm{~V}$ :

$$
\begin{gathered}
I=\frac{W}{V} \\
I=\frac{3.46 k W}{12 \mathrm{~V}} \\
I=287.74 \mathrm{~A}
\end{gathered}
$$

Asumiendo que cada arranque dure un segundo, y que el número de estos procedimientos, $\mathrm{z}$, sea el indicado en el ciclo NEDC, el consumo de corriente en el tiempo, expresado en $\mathrm{Ah}$, será:

$$
\begin{gathered}
I_{a}=I / 3600 \\
I_{a}=287.74 \mathrm{~A} / 3600 \\
I_{a}=0.08 \mathrm{Ah} \\
I_{p}=I_{a} * Z \\
I_{p}=0.08 \mathrm{Ah} * 32 \\
I=2.56 \mathrm{Ah}
\end{gathered}
$$

Este dato debe sumarse a la capacidad de la batería actual del vehículo, que en el Hyundai Getz 1.6 es 55 Ah, para determinar la capacidad de reserva de la nueva batería.

$$
\text { Capacidad }_{2}=57.45 \mathrm{Ah}
$$

Dado que no se dispone comercialmente de una batería de esta capacidad, se selecciona el valor próximo existente. Localmente existen baterías de 60Ah, y se seleccionó una de la marca EXIWILL.

Las pruebas de arranque se hicieron aproximadamente 6 meses después de instalar la nueva batería, de este tiempo, durante casi un mes se hicieron pruebas con el sistema instalado, funcionando en ciudad y en vacío. La comprobación con el probador de baterías se realizó únicamente con el vehículo detenido. En la figura 3 se muestra el resultado de la primera prueba.

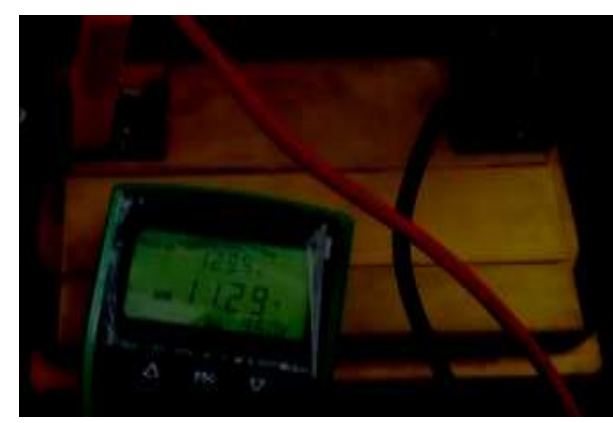

Figura 2: Primer resultado de la prueba de arranque
Comparando este resultado con los datos proporcionados con el dispositivo se puede deducir que la batería se encuentra en buen estado. En la tabla 2 se encuentra el resumen de las demás pruebas:

Tabla 2: Pruebas de arranque con la batería seleccionada

\begin{tabular}{|c|c|c|}
\hline Medición $(\mathbf{V})$ & Estado & Observación \\
\hline 11.29 & Buena & \\
\hline 11.40 & Buena & \\
\hline 11.10 & Buena & El radio se apagó \\
\hline 11.29 & Buena & \\
\hline 10.97 & Buena & El radio se apagó \\
\hline 11.24 & Buena & \\
\hline 11.57 & Buena & \\
\hline 11.57 & Buena & \\
\hline 11.48 & Buena & \\
\hline 11.49 & Buena & \\
\hline 10.94 & Buena & El radio se apagó \\
\hline 11.30 & Buena & El radio se apagó \\
\hline
\end{tabular}

La realización de estas pruebas demuestra que la selección de la batería ha sido adecuada, dado que la caída de tensión durante el arranque nunca desciende a un valor que no sea bueno, pese a que cuando desciende de $11.30 \mathrm{~V}$ se siente como algunos de los consumidores, como el radio, tienen un comportamiento diferente al normal.

En la gráfica de la figura 3 se puede apreciar la variación de los resultados en el tiempo:

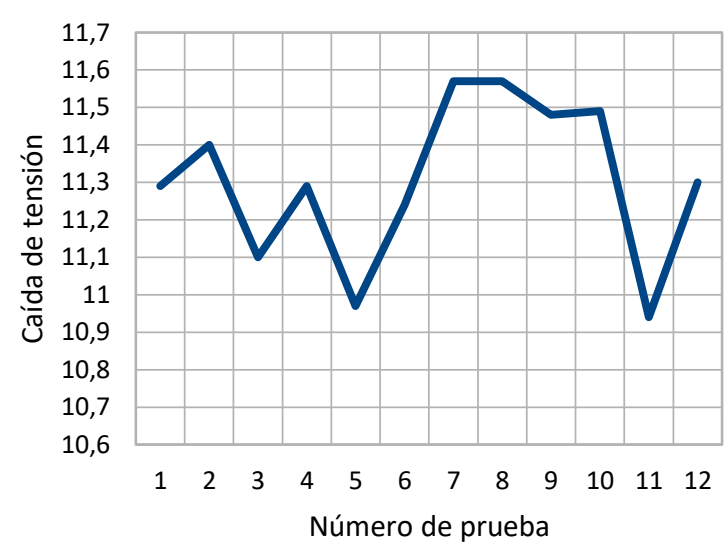

Figura 3: Variación de la tensión durante la prueba de arranque

Se puede apreciar cómo después de transcurrido un tiempo la caída de tensión durante el arranque es más 
uniforme, a pesar de que cerca del final de la prueba existe un último valor que no sigue la tendencia.

\section{CONCLUSIONES}

El cálculo de la demanda adicional de corriente que provoca el uso del sistema Start-stop, permitió seleccionar una que cuya capacidad no se vea afectada de durante el uso continuo del motor de arranque.

El nivel de tensión de la batería no desciende por debajo del nivel de tensión señalado como "bueno" por el fabricante, al realizar el proceso de arranque de forma repetitiva, a pesar de haber presentado algunos inconvenientes con el funcionamiento de otros elementos que utilizan la energía del acumulador.

El tiempo de recuperación entre cada arranque, tiempo que el motor estuvo encendido más el tiempo que estuvo apagado, si influyó en el nivel de caída de tensión de la batería, pues este valor era alcanzaba un valor más bajo cuando el lapso antes de activarse era menor.

El sistema de carga respondió de manera adecuada ante el aumento del consumo de corriente, proporcionando suficiente energía durante el lapso en que el motor se mantenía encendido, para recargar la batería hasta el momento en que se detuviera de nuevo el motor.

\section{REFERENCIAS}

[1] Ozdemir, A., Mugan, A. (2013). Stop/Start System Integration to Diesel Engine and System Modelling \& Validation, Istanbul Technical University.

[2] Bent, E., Shayler, P., La Rocca, A. (2011). The effectiveness of stop-start and thermal management measures to improve fuel economy. University of Nottingham.

[3] Universidad Tecnológica Equinoccial - Santo Domingo (2011). Estudio del motor de arranque.

[4] Rumiguano, C., Aguilar, J. (2017). Investigación de la eficiencia energética en relación al consumo de combustible y emisiones al implementar el sistema startstop en el vehículo Hyundai Getz 1.6, Universidad de las Fuerzas Armadas - ESPE.

[5] Gonzáles, M., Mas, J., Vidal, F. (2014). Sistemas de seguridad y confortabilidad. España: EDITEX.

[6] Hartman, J. (2013). How to Tune and Modify Engine Management System (Motorbooks Workshop)". Texas: Motorbooks.

[7] Hella. (2016). Intelligent battery sensor (IBS) $12 \mathrm{~V}$. Lippstad: HELLA KGaA Hueck \& Co.

[8] Horst, B.(2012).Baterías de arranque . 1era edición. Reverté.

[9] Kremer, M. (2011). In-market Application of Start-Stop Systems in European Market, FEV Project Report.

[10] Kuznetzov, M. (1967). Fundamentos de Electrotecnia. Moscú: MIR.

[11] Jaimes, E., López, R., Rico, F. (2001). Daños a la salud por contaminación atmosférica. México; D.F.: VAMEX 